\title{
SYNCHRONIZATION IN ENSEMBLES OF COUPLED MAPS WITH A MAJOR ELEMENT
}

IRYNA OMELCHENKO, YURI MAISTRENKO, AND ERIK MOSEKILDE

Received 16 March 2005

The paper investigates the conditions for full and partial synchronization in systems of coupled chaotic maps that include the presence of a major element, that is, an element that interacts with all the other elements of the system. We consider a system which consists of two globally coupled populations of one-dimensional maps that interact via a major element. The presence of this element can induce synchronization in both of the globally coupled populations even though they operate in different states. If a parameter mismatch is introduced between two populations of uncoupled maps, the presence of a major element is found to provide for the existence of states in which peripheral elements with different parameter values display similar dynamics.

\section{Introduction}

The cooperative behavior in systems of coupled nonlinear oscillators is of significant interest in many areas of science and technology [22, 27]. Traditionally, two specific and highly symmetric coupling structures have attracted most attention.

In coupled map lattices $[1,14,37]$, each oscillator is considered to interact with its nearest neighbors. Depending on the type of oscillators, the coupling strength, the dimension of the lattice, and the assumed boundary conditions, this coupling typically produces various forms of wavelike structures. In the biological realm, this situation may represent a group of interacting pancreatic $\beta$-cells [3] where the communication occurs via gap-junctional coupling. Coupled oscillator lattices have also been used as models of interacting Josephson junctions [36] and of simple forms of turbulence [7].

In populations of globally coupled maps $[15,16]$, each oscillator is considered to interact with all the other oscillators on an even basis. If the coupling is strong enough and the microscopic parameter dispersion sufficiently small, this provides for a state of coherence $[10,38]$. As the coupling strength is reduced, the coherent state is typically found to break up into clusters of oscillators that maintain internal synchronization, but have lost their synchronization with the other clusters $[26,28,29,30]$. In the presence of significant microscopic disorder, one can observe the phenomenon of oscillator death $[4,11]$. A suspension of yeast cells that interact via the metabolic products they release to 
the intercellular fluid represents an example of a globally coupled oscillator system [9]. A group of nerve cells in the brain may also act as a system of globally coupled oscillators $[33,35]$, since each cell communicates with many other nerve cells, on short as well as on long distances.

The introduction of small-world models [31, 32] has opened up for a broader discussion of the role of the coupling structure in systems of coupled chaotic oscillators. In a small-world system, one typically combines a nearest neighbor coupling with a few more or less randomly chosen interactions between elements that are farther apart. This captures to some extent the heterogeneous coupling structure that one finds in many social (and biological) systems.

The purpose of the present paper is to study different forms of full and partial synchronization in systems of chaotic oscillators that involve a major element, that is, an element that couples directly to all the other oscillators of the system. Structures of this type are characteristic of certain neuronal networks in which the so-called septo hippocampal region (supposed to act as the major element) interacts with other neurons in the brain. Besides displaying its own dynamics, the major element also experiences a component of the mean field dynamics for the oscillator population. We examine how the interaction between two populations of globally coupled maps via a major element can lead to different forms of partial synchronization.

Full synchronization, where all the elements of an ensemble display the same temporal behavior, can be achieved for identical maps [12]. In this case the motion of the system is restricted to an invariant subspace of the phase space and in this subspace it is governed by the one-dimensional map that describes the dynamics of the individual element. Partial synchronization (or clustering) occurs in coupled map ensembles when the population of maps splits into subgroups (clusters) with different dynamics, but such that all elements within a given cluster move in synchrony. In the cluster state, the motion of the system is also restricted to an invariant manifold in the phase space $[5,6]$, the dimension of this manifold being given by the number of clusters in the system.

The largest transverse Lyapunov exponent is a measure of the stability for the synchronized chaotic state in the direction perpendicular to the synchronization manifold. If this exponent is negative, all trajectories in the neighborhood of the synchronized state will be attracted on the average by the synchronized state. The transition in which the largest transverse Lyapunov exponent becomes positive, and transverse stability is lost, is often referred as a blowout bifurcation [23].

In order to understand the influence of the coupling structure on the synchronization properties, let us shortly review some of the properties of the coupled map system

$$
\begin{gathered}
x_{i}^{t+1}=(1-\varepsilon) f\left(x_{i}^{t}\right)+\varepsilon f\left(z^{t}\right), \quad i=1, \ldots, N, \\
z^{t+1}=(1-\varepsilon) f\left(z^{t}\right)+\frac{\varepsilon}{N} \sum_{j=1}^{N} f\left(x_{j}^{t}\right),
\end{gathered}
$$

where $\left(x_{1}, \ldots, x_{N}, z\right) \in \mathbb{R}^{N+1}, t=0,1, \ldots$ is a discrete time index. $f: \mathbb{R} \rightarrow \mathbb{R}$ is a smooth one-dimensional map, for which we will use the logistic map $f(x)=f_{a}(x)=\operatorname{ax}(1-x)$. System (1.1) consists of a population of $N$ identical noninteracting elements $x_{i}$ and of 


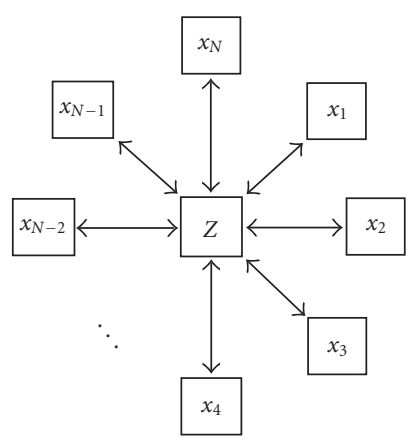

(a)

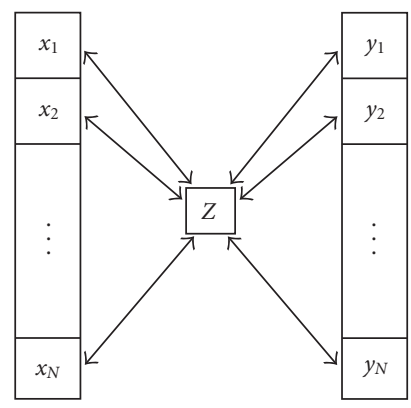

(b)

Figure 1.1. Schematic form of the considered models. (a) Star system; (b) Two globally coupled groups with a major element. In both schemes squares denote individual elements of the system, arrows denote bidirectional couplings, and $z$ is the major element. In (b) elements arranged in a column belong to a globally coupled population.

the major element $z$, which is coupled to each of the elements $x_{i}$ with the same coupling strength. The parameter $\varepsilon$ controls the coupling strength. The coupling structure of system (1.1) is illustrated in Figure 1.1a. This so-called star topology represents one of the well-studied prototypes of graph theory [13].

System (1.1) allows for the existence of the fully synchronized state $C=\left\{x_{1}=\cdots=\right.$ $\left.x_{N}=z\right\}$ and of the two-cluster state $C_{2}=\left\{x_{1}=x_{2}=\cdots=x_{N} \neq z\right\}$, where all elements $x_{i}$ are synchronized with each other, but not synchronized with the major element $z$.

System (1.1) always has $N+1$ degrees of freedom. Assume that full synchronization takes place in the system. The dynamics is then restricted to a one-dimensional invariant manifold in phase space and, therefore, the Jacobian matrix has one eigendirection along this manifold and $N$ eigendirections transverse to it. Trajectories of the system can lose their transverse stability in $N$ directions.

For any $N$, the Jacobian matrix for the fully synchronized system, where $\mathbf{x}:=x_{1}=$ $\cdots=x_{N}=z$, has three different eigenvalues: $\nu_{\|}=f^{\prime}(\mathbf{x}), \nu_{\perp, 1}=(1-\varepsilon) f^{\prime}(\mathbf{x}), \nu_{\perp, 2}=(1-$ $2 \varepsilon) f^{\prime}(\mathbf{x})$. The eigenvalues $\nu_{\|}$and $\nu_{\perp, 2}$ have multiplicities 1 , and the eigenvalue $v_{\perp, 1}$ has multiplicity $N-1$.

The transverse stability of the fully synchronized state is controlled by the transverse Lyapunov exponents:

$$
\lambda_{\perp, 1}=\lim _{k \rightarrow \infty} \frac{1}{k} \sum_{n=0}^{k-1} \ln \left|f^{\prime}\left(x^{n}\right)(1-\varepsilon)\right|, \quad \lambda_{\perp, 2}=\lim _{k \rightarrow \infty} \frac{1}{k} \sum_{n=0}^{k-1} \ln \left|f^{\prime}\left(x^{n}\right)(1-2 \varepsilon)\right|,
$$

where $\left\{x^{n}\right\}_{n=1}^{\infty}$ is a typical trajectory of the one-dimensional map $f_{a}(x)$.

Note, that a negative value of the transverse Lyapunov exponents implies weak stability, as defined by Milnor [21]. This means that the coherent (chaotic) state is attracting on the average. However, a negative Lyapunov exponent does not imply asymptotic 


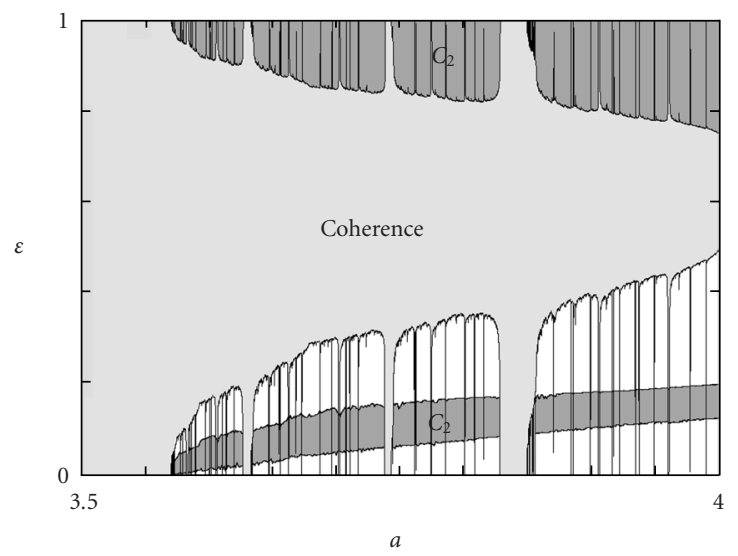

Figure 1.2. Stability diagram for the star system (1.1). $a$ is the nonlinearity parameter of the individual logistic map, and $\varepsilon$ is the coupling strength. The region of transverse stability for full synchronization (coherence) is shown light gray; the regions of stability for the two-cluster state $C_{2}$ are dark gray. White zones correspond to states of desynchronization in the system. The diagram applies for any number of elements in system (1.1).

stability. Any $(N+1)$-dimensional neighborhood of the synchronized state may contain initial conditions from which the trajectories approach other limiting states or diverge to infinity [2].

If the stability of the synchronized state is lost because $\lambda_{\perp, 2}$ becomes positive, we arrive at the two-cluster state $C_{2}$. This state is sometimes referred to as the "drum-head" mode $[24,25]$. In the state $C_{2}$, the dynamics of the $(N+1)$-dimensional system $(1.1)$ is restricted to a two-dimensional invariant manifold in phase space and governed there by the system

$$
\begin{aligned}
x^{t+1} & =(1-\varepsilon) f\left(x^{t}\right)+\varepsilon f\left(z^{t}\right), \\
z^{t+1} & =(1-\varepsilon) f\left(z^{t}\right)+\varepsilon f\left(x^{t}\right) .
\end{aligned}
$$

Systems of the form (1.3) have been studied in detail by Popovych et al. [28, 29], and many of their results may be found in the recent book by Mosekilde et al. [22]

The stability of the two-cluster state $C_{2}$ can be lost in $N-1$ transverse directions and is controlled by $N-1$ transverse Lyapunov exponents, which all are equal to $\lambda_{\perp, 1}$. They correspond to different ways of breaking of the synchronized population of $N$ maps $x_{1}, \ldots, x_{N}$ into two parts.

From the above analysis it is obvious, that the stability regions of fully and partially synchronized states in the star system (1.1) are independent of the size of the system. Figure 1.2 shows the stability regions of $C$ and $C_{2}$ in the parameter plane $(a, \varepsilon)$ where, as before, $a$ is the nonlinearity parameter of single map $f_{a}(x)=\operatorname{ax}(1-x)$.

In the present paper, we propose various modifications of the star system and examine their properties. First, we consider a population of identical maps that consists of two globally coupled groups each of $N$ elements. Moreover, each individual map will be coupled to the major element, which is shared between both groups. The system describing 
this situation has the form:

$$
\begin{aligned}
x_{i}^{t+1} & =(1-\varepsilon) f\left(x_{i}^{t}\right)+\frac{\varepsilon}{N+1}\left(\sum_{j=1}^{N} f\left(x_{j}^{t}\right)+f\left(z^{t}\right)\right), \\
y_{i}^{t+1} & =(1-\varepsilon) f\left(y_{i}^{t}\right)+\frac{\varepsilon}{N+1}\left(\sum_{j=1}^{N} f\left(y_{j}^{t}\right)+f\left(z^{t}\right)\right), \quad i=1, \ldots, N, \\
z^{t+1} & =(1-\varepsilon) f\left(z^{t}\right)+\frac{\varepsilon}{2 N} \sum_{j=1}^{N}\left(f\left(x_{j}^{t}\right)+f\left(y_{j}^{t}\right)\right),
\end{aligned}
$$

where $\left\{x_{i}\right\}_{i=1}^{N}$ and $\left\{y_{i}\right\}_{i=1}^{N}$ are two identical ensembles each of $N$ globally coupled maps, and $z$ is the major element, which is coupled to all of the $2 N$ maps. $\varepsilon$ is the map-tomap coupling strength in the system. The local one-dimensional map we again choose to be the logistic map $f(x)=f_{a}(x)=a x(1-x)$. The coupling structure of this system is shown in Figure 1.1b. Here, elements connected in columns represent globally coupled ensembles, $z$ is the major element, and arrows denote bidirectional couplings between major and peripheral elements in the system.

It is well-known that the parameter regions of stability for fully and partially synchronized states in the ensemble of globally coupled logistic maps are rather small [15]. In previous works, we considered two identical globally coupled ensembles with additional pairwise couplings between their elements $[19,20]$. This structure results in wider parameter regions of stability for the fully and partially synchronized states.

As mentioned above, the structures of these systems derive from the form of certain neuronal networks in which the septo hippocampal region interacts with other neurons in the brain $[8,17,18]$. The star system (1.1) and its modification with parameter mismatch can be considered as simplified models for such neuronal networks. It is wellknown that the brain contains so-called cortical columns of neurons, where the neuron interaction is stronger than the interaction between neurons in different columns. Hence, system (1.4) can be interpreted as two cortical columns, represented by globally coupled groups, interacting via the septo hippocampal region, represented by the major element. A better understanding of the synchronization properties of such systems is essential for the use of deep brain stimulation techniques to treat various forms of tremor [34].

\section{Two globally coupled groups interacting via a major element}

Let us now turn our attention to system (1.4). Our purpose is to examine how the presence of the major element influences the stability properties and to find out if it is possible to reach a state of synchronization for the maps $\left\{x_{i}\right\}$ and $\left\{y_{i}\right\}$, which are connected only through the coupling via the major element $z$.

The coupling structure of system (1.4) allows for the existence of invariant manifolds in phase space corresponding to the following states:

(i) Full synchronization $C: \underbrace{x_{1}=\cdots=x_{N}=y_{1}=\cdots=y_{N}=z}_{\text {xyz }}$. 
244 Coupled maps with a major element: synchronization

(ii) Two-cluster state $C_{2}: \underbrace{x_{1}=\cdots=x_{N}=y_{1}=\cdots=y_{N}}_{\mathbf{x y}} \neq z$.

(iii) Three-cluster state $C_{3}: \underbrace{x_{1}=\cdots=x_{N}}_{\mathbf{x}} ; \underbrace{y_{1}=\cdots=y_{N}}_{\mathbf{y}} ; \mathbf{x} \neq \mathbf{y}, \mathbf{x} \neq z, \mathbf{y} \neq z$.

System (1.4) has always $2 N+1$ degrees of freedom. The number of transverse directions of potential instability for the synchronized states depend directly on the number of clusters in the considered state. Increasing the number of clusters reduces the number of transverse instability directions. If we consider the transition $C \rightarrow C_{2} \rightarrow C_{3}$, we observe an increasing number of clusters: $1 \rightarrow 2 \rightarrow 3$, and a corresponding decreasing number of directions of possible transverse instabilities: $2 N \rightarrow(2 N-1) \rightarrow(2 N-2)$. Below we consider the eigensystems of the Jacobian matrix of system (1.4) for the various cases in more detail.

Let us first analyse the three-cluster state

$$
C_{3}:\{\underbrace{x_{1}=\cdots=x_{N}}_{\mathbf{x}} ; \underbrace{y_{1}=\cdots=y_{N}}_{\mathbf{y}} ; \mathbf{x} \neq \mathbf{y}, \mathbf{x} \neq z, \mathbf{y} \neq z\},
$$

where elements of each globally coupled group are synchronized inside the group, but there is no synchronization neither with the major element nor between the groups.

The eigenvalues of the Jacobian matrix $J$ can be divided into two types. Three of them coincide with the eigenvalues of the Jacobian matrix of the three-dimensional system

$$
\begin{aligned}
& x^{t+1}=\left(1-\frac{\varepsilon}{N+1}\right) f\left(x^{t}\right)+\frac{\varepsilon}{N+1} f\left(z^{t}\right), \\
& y^{t+1}=\left(1-\frac{\varepsilon}{N+1}\right) f\left(y^{t}\right)+\frac{\varepsilon}{N+1} f\left(z^{t}\right), \\
& z^{t+1}=(1-\varepsilon) f\left(z^{t}\right)+\frac{\varepsilon}{2}\left(f\left(x^{t}\right)+f\left(y^{t}\right)\right) .
\end{aligned}
$$

This system describes the in-cluster dynamics of system (1.4) in the three-dimensional manifold, corresponding to the cluster state $C_{3}$.

The other $2 \mathrm{~N}-2$ eigenvalues of the Jacobian matrix $J$ correspond to the transverse eigendirections:

$$
u_{\mathrm{x}}=(\xi_{1}, \ldots, \xi_{N}, \underbrace{0, \ldots, 0}_{N}, 0), \quad \sum_{i=1}^{N} \xi_{i}=0, \quad u_{\mathrm{y}}=(\underbrace{0, \ldots, 0}_{N}, \eta_{1}, \ldots, \eta_{N}, 0), \quad \sum_{i=1}^{N} \eta_{i}=0 .
$$

The corresponding transverse Lyapunov exponents are

$$
\begin{aligned}
& \lambda_{\mathbf{x}}=\lim _{k \rightarrow \infty} \frac{1}{k} \sum_{n=0}^{k-1} \ln \left|f^{\prime}\left(x^{n}\right)(1-\varepsilon)\right|, \\
& \lambda_{\mathbf{y}}=\lim _{k \rightarrow \infty} \frac{1}{k} \sum_{n=0}^{k-1} \ln \left|f^{\prime}\left(y^{n}\right)(1-\varepsilon)\right|,
\end{aligned}
$$


where $\left\{\left(x^{n}, y^{n}, z^{n}\right)\right\}_{n=0}^{\infty}$ is a typical trajectory of system (2.2). From the form of the transverse eigenvectors it is clear that the multiplicity of $\lambda_{\mathbf{x}}\left(\lambda_{\mathbf{y}}\right)$ is $N-1$, and each of these exponents corresponds to breaking of the synchronized group of $N$ globally coupled elements. The three-cluster state $C_{3}$ is transversely stable when all its transverse Lyapunov exponents are negative.

Figure 2.1 shows the borders of the transverse stability regions for the three-cluster state $C_{3}$ in system (1.4) with $N=10$ (bold curve) and $N=100$ (thin curve). For values of the coupling parameter above these curves, the three-cluster state $C_{3}$ is transversely stable. As our calculations show, these regions do not vary much with the number of elements in the ensemble. It is easy to see from Figure 2.1, that increasing $N$ only results in a small decrease of the size of the stability regions.

Consider now the two-cluster state

$$
C_{2}:\{\underbrace{x_{1}=\cdots=x_{N}=y_{1}=\cdots=y_{N}}_{\mathbf{x y}} \neq z\},
$$

where all the peripheral elements are equal and compose one cluster, while the major element forms a separate cluster. In this state two clusters $\mathbf{x}$ and $\mathbf{y}$ of $C_{3}$ overlap $(\mathbf{x}=\mathbf{y})$ and form a single cluster $\mathbf{x y}$.

The dynamics of system (1.4) is restricted to a two-dimensional manifold and governed there by the two-dimensional system:

$$
\begin{gathered}
x^{t+1}=\left(1-\frac{\varepsilon}{N+1}\right) f\left(x^{t}\right)+\frac{\varepsilon}{N+1} f\left(z^{t}\right), \\
z^{t+1}=(1-\varepsilon) f\left(z^{t}\right)+\varepsilon f\left(x^{t}\right) .
\end{gathered}
$$

The Jacobian matrix of system (1.4) has two eigenvalues, which coincide with the eigenvalues of system (2.6). The corresponding eigenvectors are:

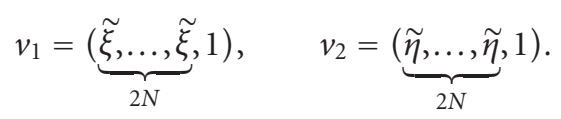

Besides $2 N-2$ transverse eigendirections $u_{\mathrm{x}}$ and $u_{\mathrm{y}}$, there appears one additional transverse eigenvector:

$$
u_{\mathrm{xy}}=(\underbrace{-1, \ldots,-1}_{N} \underbrace{1, \ldots, 1}_{N}, 0)
$$

with the corresponding transverse Lyapunov exponent:

$$
\lambda_{\mathrm{xy}}=\lim _{k \rightarrow \infty} \frac{1}{k} \sum_{n=0}^{k-1} \ln \left|f^{\prime}\left(x^{n}\right)\left(1-\frac{\varepsilon}{N+1}\right)\right|,
$$

now evaluated for a typical trajectory $\left\{\left(x^{n}, z^{n}\right)\right\}_{n=0}^{\infty}$ of system (2.6). From the form of the eigenvector $u_{\mathrm{xy}}$ we conclude that the Lyapunov exponent $\lambda_{\mathrm{xy}}$ corresponds to breaking of the cluster $\mathbf{x y}$ of $2 N$ elements into two groups $\mathbf{x}$ and $\mathbf{y}$ of $N$ elements each. When $\lambda_{\mathbf{x y}}$ 


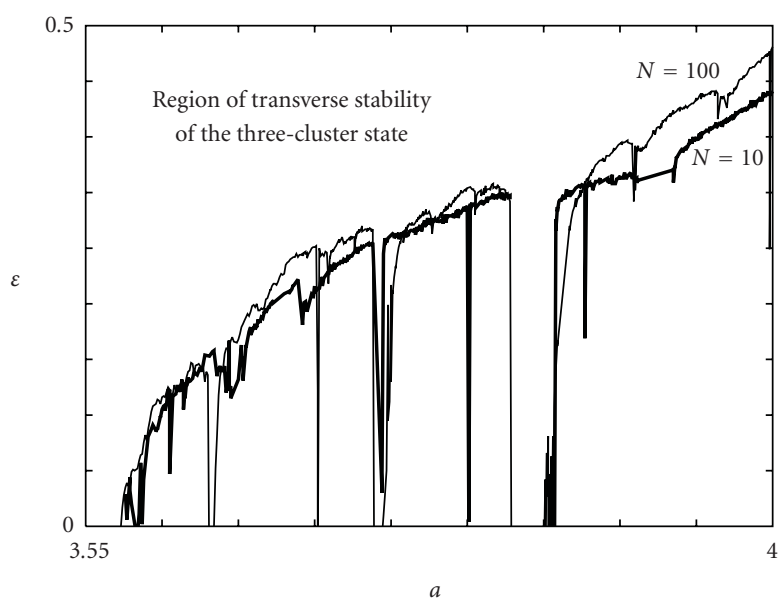

Figure 2.1. Borders of the region of transverse stability for the three-cluster state $C_{3}$ in system (1.4) with $N=10$ (bold curve) and $N=100$ (thin curve). Above these curves $C_{3}$ is transversely stable. As before, $a$ is the nonlinearity parameter of the individual map, and $\varepsilon$ is the coupling strength in the system.

becomes positive (while the Lyapunov exponents $\lambda_{\mathbf{x}}$ and $\lambda_{\mathbf{y}}$ are negative), we observe a transition from $C_{2}$ to the three-cluster state $C_{3}$.

For $C_{2}$, as $\mathbf{x}=\mathbf{y}$, the Lyapunov exponents $\lambda_{\mathbf{x}}$ and $\lambda_{\mathbf{y}}$ merge into a single exponent of multiplicity $2 N-2$. Therefore, we have totally $2 N-1$ possible directions of transverse instability. The two-cluster state $C_{2}$ is transversely stable when its transverse Lyapunov exponents $\lambda_{\mathbf{x y}}$ and $\lambda_{\mathbf{x}}\left(=\lambda_{\mathbf{y}}\right)$ are negative.

Calculation of the above transverse Lyapunov exponents for different $N$ shows, that the regions in parameter space where they are both negative do not overlap. Moreover, increasing $N$ increases the distance between the parameter regions where $\lambda_{\mathbf{x y}}$ and $\lambda_{\mathbf{x}}$ are negative. We conclude that the two-cluster state $C_{2}$ can not be stabilized.

Finally, consider the coherent state where all the variables display identical temporal behavior: $C=\left\{x_{1}=\cdots=x_{N}=y_{1}=\cdots=y_{N}=z\right\}$. In this state, the dynamics of system (1.4) is restricted to the main diagonal $D_{2 N+1}=\left\{\mathbf{w} \in \mathbb{R}^{2 N+1} \mid w_{1}=\cdots=w_{2 N+1}\right\}$ of the $(2 N+1)$-dimensional phase space, and on this diagonal the motion is governed by the one-dimensional map $f_{a}(x)$.

Transverse stability of the synchronized state can be lost in the $2 N-1$ directions, defined by the eigenvectors $u_{\mathrm{x}}, u_{\mathrm{y}}, u_{\mathrm{xy}}$, and in the direction of the eigenvector

$$
u_{\mathrm{xyz}}=(\underbrace{-1 /(N+1), \ldots,-1 /(N+1)}_{2 N}, 1) .
$$

Totally we have $2 \mathrm{~N}$ directions of transverse instability of the fully synchronized state in system (1.4). The coherent state $C$ is transversely stable when the Lyapunov exponents for all of these $2 \mathrm{~N}$ transverse directions are negative. 
The transverse Lyapunov exponent corresponding to the eigendirection $u_{\mathrm{xyz}}$ has a form

$$
\lambda_{\mathrm{xyz}}=\lim _{k \rightarrow \infty} \frac{1}{k} \sum_{n=0}^{k-1} \ln \left|f^{\prime}\left(x^{n}\right)\left(1-\frac{(N+2) \varepsilon}{N+1}\right)\right| .
$$

When $\lambda_{\text {xyz }}$ becomes positive, we observe a transition from the coherent state $C$ to the twocluster state $C_{2}$. The corresponding eigenvector of the Jacobian matrix is $\bar{u}=(1,1, \ldots, 1)$. It is directed along the main diagonal $D_{2 N+1}$ of the $(2 N+1)$-dimensional phase space of the system. The associated Lyapunov exponent defines the stability of the trajectories of the system along the diagonal and coincides with the Lyapunov exponent for the single one-dimensional map $f_{a}(x)$.

Obviously, the stability regions for system (1.4) depend not only on the nonlinearity parameter $a$ of the local map, but also on the number of maps $N$ in each group. We recall that the star system (1.1) showed no such dependence on $N$.

The regions of stability for the coherent state of system (1.4) with $N=2,4,10$ are displayed in Figure 2.2. This figure shows how even small increases of the number of elements in the system give rise to essential changes of the coherence stability region. Figure 2.2a shows the region of transverse stability for the coherent state with $N=2$. Note, that in this case the region of stability is an empty set for $a=4$. It is easy to see from Figures $2.2 \mathrm{~b}$ and $2.2 \mathrm{c}$ that with increasing $N$, broader and broader intervals for $a$ develop in which we do not have any region of stability. Hence, the region of stability for the coherent state may be broken into separate intervals. Moreover, these stable intervals typically correspond to parameter values, where the local map $f_{a}(x)$ displays a periodic behavior. In Figure 2.2c, for instance, $a^{\prime} \cong 3.83 \ldots, a^{\prime \prime} \cong 3.63 \ldots, a^{*} \cong 3.569 \ldots$, and the largest stability regions appear near these values where the one-dimensional logistic map $f_{a}(x)$ displays simple low-periodic behavior.

For values of the nonlinearity parameter $a$ corresponding to periodic windows of the individual map $f_{a}(x)$, the coherent state is transversely stable at any value of the coupling strength. Therefore there are also an infinite number of thin regions of stability, corresponding to each of the periodic windows. Figure 2.2 shows only the largest regions.

It is obvious from Figure 2.2, that a state of full synchronization in system (1.4) can be realized only for small numbers of elements in the system.

\section{Star system with parameter mismatch}

In the previous section, we considered a system where the dynamics of every individual element was governed by logistic maps with the same nonlinearity parameter. Let us now introduce a modification of system (1.1) by which the peripheral elements are divided into two groups with different nonlinearity parameters, while the major element is governed by the logistic map with a third nonlinearity parameter.

We will consider the symmetric case, where the two groups of peripheral elements have the same number of elements. Let $N$ be an even number, $N=2 K$, then the star system 


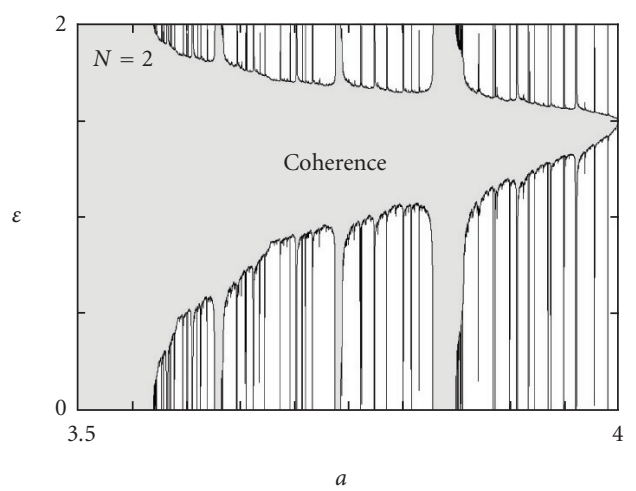

(a)

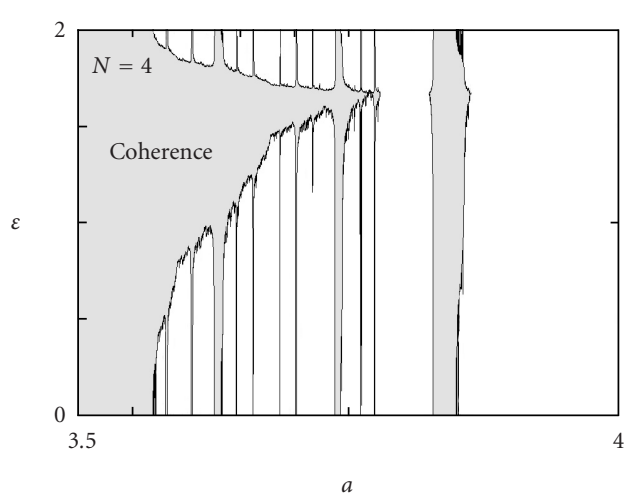

(b)

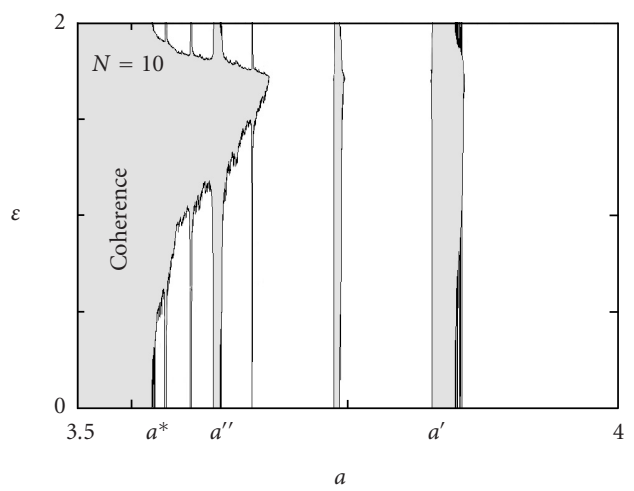

(c)

Figure 2.2. Parameter regions of transverse stability for the coherent state in system (1.4) with (a) $N=2$; (b) $N=4$; and (c) $N=10$. Note, as shown in (c), how the stability regions with increasing $N$ concentrate at values of the nonlinearity parameter that correspond to periodic behavior of the one-dimensional logistic map. 


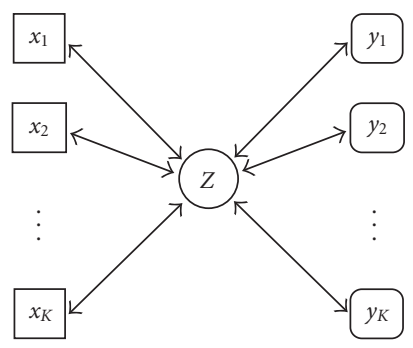

Figure 3.1. Structure of system (3.1) shown schematically. The major element is denoted $z$ and the peripheral elements are denoted $x_{i}$ and $y_{i}, i=1, \ldots, K$. Different shapes mean that the local dynamics of the elements are governed by one-dimensional maps with different nonlinearity parameters. Arrows denote bidirectional couplings.

with the assumed mismatch will have the form:

$$
\begin{aligned}
x_{i}^{t+1} & =(1-\varepsilon) f_{a_{1}}\left(x_{i}^{t}\right)+\varepsilon f_{a}\left(z^{t}\right), \\
y_{i}^{t+1} & =(1-\varepsilon) f_{a_{2}}\left(y_{i}^{t}\right)+\varepsilon f_{a}\left(z^{t}\right), \quad i=1, \ldots, K, \\
z^{t+1} & =(1-\varepsilon) f_{a}\left(z^{t}\right)+\frac{\varepsilon}{2 K} \sum_{j=1}^{K}\left(f_{a_{1}}\left(x_{j}^{t}\right)+f_{a_{2}}\left(y_{j}^{t}\right)\right),
\end{aligned}
$$

where $f_{q}(x)=q x(1-x), t=0,1, \ldots$, and $\varepsilon$ is the coupling strength. The structure of system (3.1) is shown schematically in Figure 3.1. Here, $z$ is the major element, $x_{i}$ and $y_{i}$, $i=1, \ldots, K$, are peripheral elements, and arrows denote bidirectional couplings. The different shapes of the elements symbolize that the dynamics is governed by maps with different nonlinearity parameters.

Full synchronization in system (3.1) where $x_{1}^{t}=\cdots=x_{K}^{t}=y_{1}^{t}=\cdots=y_{K}^{t}=z^{t}$ is impossible because the corresponding one-dimensional manifold is not invariant under the dynamics of the system.

However, we can consider the three-cluster state

$$
\widehat{C}_{3}=\{\underbrace{x_{1}=\cdots=x_{K}}_{\hat{\mathbf{x}}} ; \underbrace{y_{1}=\cdots=y_{K}}_{\hat{\mathbf{y}}} ; z ; \hat{\mathbf{x}} \neq \hat{\mathbf{y}}, \hat{\mathbf{x}} \neq z, \hat{\mathbf{y}} \neq z\} .
$$

It is easy to check by simple substitution that the three-dimensional manifold $M_{3}^{2 K+1}=$ $\left\{\mathbf{w} \in \mathbb{R}^{2 K+1} \mid w_{1}=\cdots=w_{K} ; w_{K+1}=\cdots=w_{2 K}\right\}$ corresponding to $\hat{C}_{3}$ is invariant under the dynamics of system (3.1). The in-cluster dynamics on this manifold is governed by the three-dimensional system:

$$
\begin{aligned}
x^{t+1} & =(1-\varepsilon) f_{a_{1}}\left(x^{t}\right)+\varepsilon f_{a}\left(z^{t}\right) \\
y^{t+1} & =(1-\varepsilon) f_{a_{2}}\left(y^{t}\right)+\varepsilon f_{a}\left(z^{t}\right) \\
z^{t+1} & =(1-\varepsilon) f_{a}\left(z^{t}\right)+\frac{\varepsilon}{2}\left(f_{a_{1}}\left(x^{t}\right)+f_{a_{2}}\left(y^{t}\right)\right) .
\end{aligned}
$$


The transverse eigenvalues of the Jacobian matrix, taken in the point

$$
(\underbrace{x, \ldots, x}_{K}, \underbrace{y, \ldots, y}_{K}, z) \in \mathbb{R}^{2 K+1},
$$

are given by $\nu_{\perp, 1}=(1-\varepsilon) f_{a_{1}}^{\prime}(x), \nu_{\perp, 2}=(1-\varepsilon) f_{a_{2}}^{\prime}(y)$, and they each have multiplicity $(K-1)$. Here $x$ and $y$ are the first two coordinates of the three-dimensional state vector $(x, y, z)$ of system (3.3). The corresponding transverse Lyapunov exponents define the transverse stability of the three-cluster state $\widehat{C}_{3}$ :

$$
\begin{aligned}
& \lambda_{\perp, a_{1}}=\lim _{k \rightarrow \infty} \frac{1}{k} \sum_{n=0}^{k-1}\left|f_{a_{1}}^{\prime}\left(x^{n}\right)(1-\varepsilon)\right|, \\
& \lambda_{\perp, a_{2}}=\lim _{k \rightarrow \infty} \frac{1}{k} \sum_{n=0}^{k-1}\left|f_{a_{2}}^{\prime}\left(y^{n}\right)(1-\varepsilon)\right| .
\end{aligned}
$$

Figure 3.2 shows graphs of the transverse Lyapunov exponents $\lambda_{\perp, a_{1}}$ and $\lambda_{\perp, a_{2}}$ for the three-cluster state $\widehat{C}_{3}$ in system (3.1) with $a_{1}=4$ and $a_{2}=3.8$. The major element is chosen to be ruled by a logistic map with the nonlinearity parameter $a=4$ (Figure 3.2a) and $a=3.8$ (Figure 3.2b). Inspection of these graphs shows that four types of behavior can be observed in the system. Regions where both transverse Lyapunov exponents are negative correspond to the transverse stability of the three-cluster state $\hat{C}_{3}$, that is, when elements inside both groups are synchronized: $x_{1}=\cdots=x_{K} ; y_{1}=\cdots=y_{K}$. Regions of this type are hatched.

The second type corresponds to positive values of $\lambda_{\perp, a_{1}}$ and negative values of $\lambda_{\perp, a_{2}}$. These regions are shown light gray. In this case, elements in the group $\left\{y_{i}\right\}_{i=1}^{K}$ are synchronized, while the other group of peripheral elements are not synchronized.

The third type of behavior is opposite to the previous and appears when $\lambda_{\perp, a_{1}}<0$ and $\lambda_{\perp, a_{2}}>0$. By analogy, in this case we have synchronization in group $\left\{x_{i}\right\}_{i=1}^{K}$ and do not have it in $\left\{y_{i}\right\}_{i=1}^{K}$. Dark gray color denotes these regions.

Finally, the fourth type corresponds to complete desynchronization in the system, when $\lambda_{\perp, a_{1}}$ and $\lambda_{\perp, a_{2}}$ are both positive.

Note, that for both of the chosen values for the nonlinearity parameter of the major element, all four types of behavior can occur in the system. This is a consequence of the fact that maps with different level of nonlinearity are coupled, and this has a significant influence on their behavior. Hence, to reach the strongest effect of the presence of the major element, the local behavior of the peripheral elements should have similar values of the nonlinearity.

Figure 3.3 shows examples of the influence of the major element on the synchronization of uncoupled peripheral elements, ruled locally by different one-dimensional maps. For the examples shown, $a_{1}=4, a_{2}=3.8$, and $a=4$. In Figures 3.3a and 3.3b $\varepsilon=0.7$, and system (3.1) reaches the three-cluster state $\hat{C}_{3}$. Figure 3.3 a shows values of $y$ versus $x$ and Figure 3.3b shows values of $z$ versus $x$. From the obtained graphs it is easy to see, that the values of $x$ and $y$ are very close, and the values of $x$ and $z$, which actually are ruled 


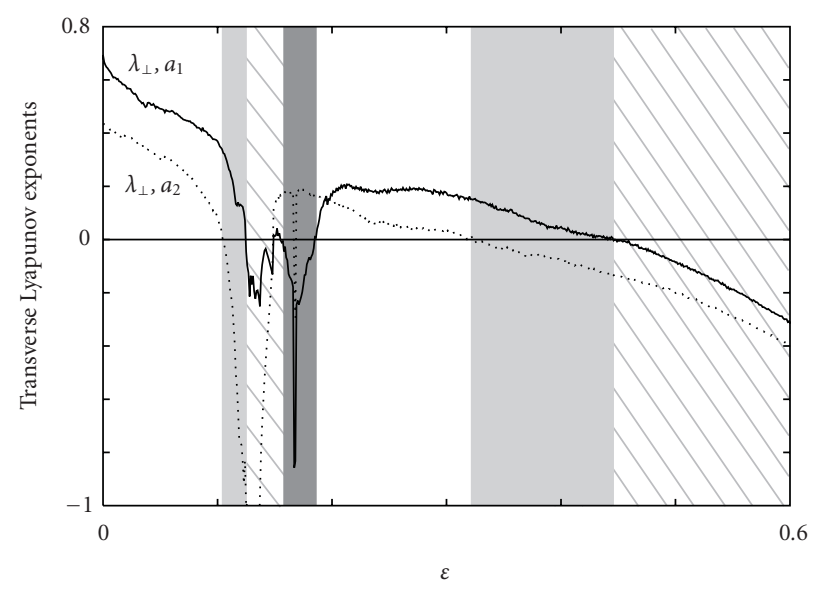

(a)

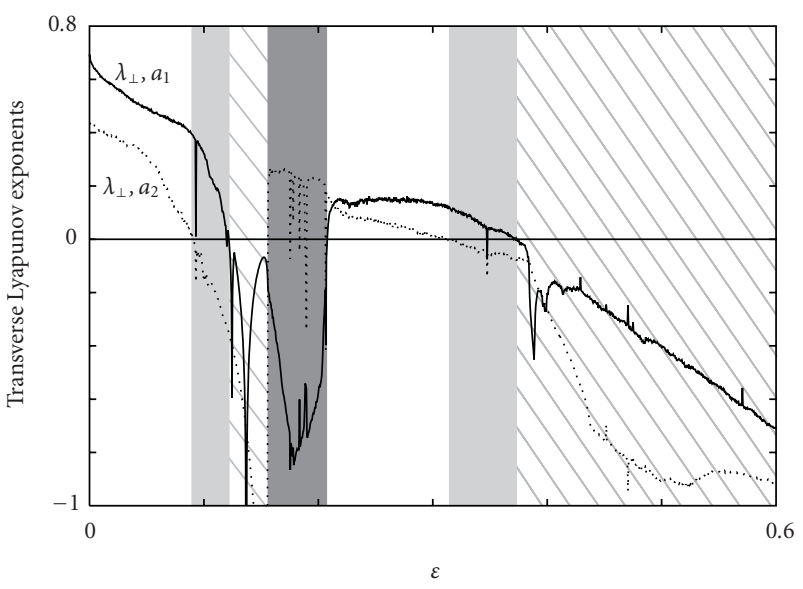

(b)

Figure 3.2. Graphs of the transverse Lyapunov exponents for the three-cluster state $\hat{C}_{3}$ in system (3.1) with (a) $a_{1}=4.0, a_{2}=3.8$, and $a=4.0$ and with (b) $a_{1}=4.0, a_{2}=3.8$, and $a=3.8$. $\lambda_{\perp, a_{1}}$ is drawn by solid line and $\lambda_{\perp, a_{2}}$ is drawn by dotted line. Hatched regions denote parameter regions of transverse stability for $\hat{C}_{3}$, where both the transverse Lyapunov exponents are negative. Light gray and dark gray regions denote parameter regions where the transverse Lyapunov exponents have different signs, $\lambda_{\perp, a_{1}}<0$ in dark gray regions, and $\lambda_{\perp, a_{2}}<0$ in light gray regions. This implies stability of the synchronized state only in one of the ensembles, $\left\{x_{i}\right\}_{i=1}^{K}$ or $\left\{y_{i}\right\}_{i=1}^{K}$, respectively.

by maps with the same nonlinearity parameter, are less close. Figures $3.3 \mathrm{c}$ and $3.3 \mathrm{~d}$ show analogous graphs for $\varepsilon=0.8$. Here, as in the previous case, the values of the peripheral elements are very close while the values of the major element are quite different. 


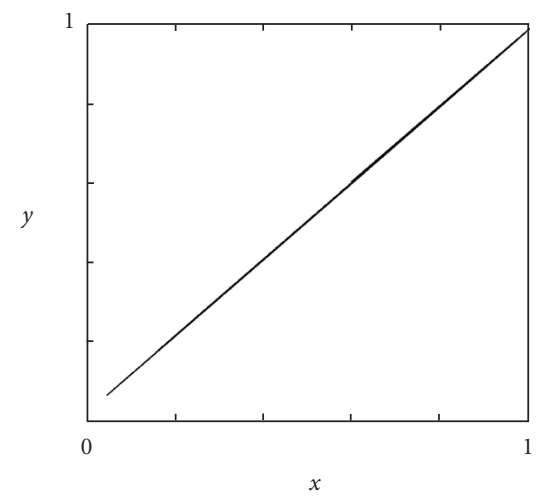

(a)

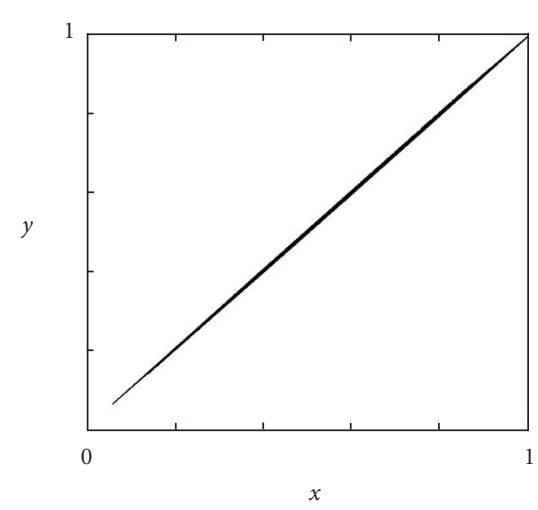

(c)

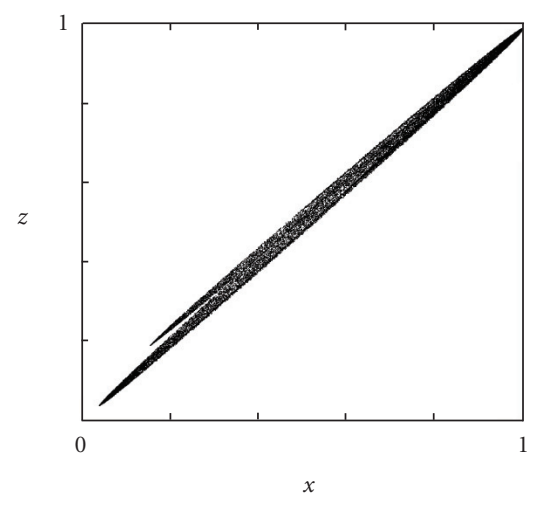

(b)

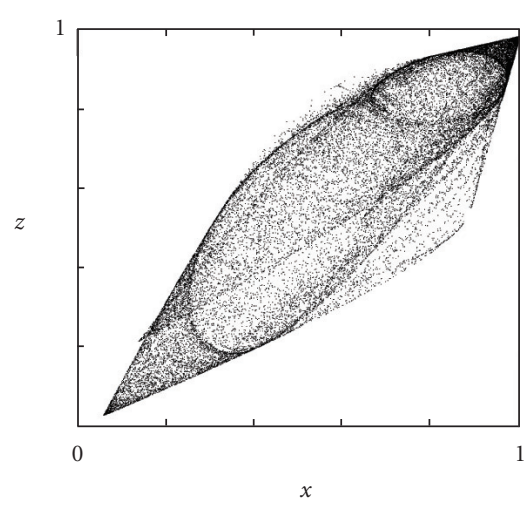

(d)

Figure 3.3. Phase-space projections for system (3.1) in three-cluster state $\hat{C}_{3}$. Nonlinearity parameters are $a_{1}=4, a_{2}=3.8$, and $a=4$. For (a) and (b) $\varepsilon=0.7$, and for (c) and (d) $\varepsilon=0.8$. (a) and (c) show the distribution of values of the elements in two groups of peripheral maps; (b) and (d) show the distribution between the major element $z$ and one of the peripheral groups.

These examples illustrate that, even when exact synchronization is not possible, the presence of a major element allows the system to reach a state, where peripheral elements with different nonlinearities demonstrate almost synchronized behavior, without being synchronized with the major element.

\section{Conclusion}

To investigate the dynamic properties of coupled map systems with a major element, we considered a modifications of the so-called star system, in which only couplings between 
the major and the individual peripheral elements take place, while the peripheral elements are mutually uncoupled. The star system with identical elements exhibits a state of full synchronization, where all the elements are synchronized with the major element, as well as a partially synchronized state, where all peripheral elements move in synchrony, but not in synchrony with the major element. The existence of this latter state illustrates the role of the major element: its presence allows synchronization to be achieved between otherwise uncoupled oscillators. The regions of transverse stability for the two synchronized states are rather large. Moreover, synchronization of peripheral elements is possible for any nonlinearity parameter of the individual logistic map. The stability regions for the star system do not depend on the number $N$ of peripheral elements.

In the system of two globally coupled groups interacting via a major element, the fully synchronized state can be stable provided that the ensemble includes a relatively small number of elements. This system exhibits a rather strong dependence of the stability regions on the number of maps. We observed that increasing $N$ produces a significant reduction of the coherence stability regions. The advantage of the coupling structure of system (1.4) appears in the large stability region for the three-cluster state $C_{3}$. This allows synchronization of elements within each of the globally coupled ensembles at parameters values, where it is impossible to achieve the synchronization for the individual globally coupled ensemble of logistic maps.

As a further development of the idea of a major element, we considered a modification of the star system with a mismatch in the nonlinearity parameters. We supposed the population of peripheral maps to consist of two groups for which the dynamics are governed by one-dimensional logistic maps with different nonlinearity parameters, and tried to tune the nonlinearity of the major element to achieve synchronization. In this case, exact synchronization of the peripheral elements is impossible, but we have observed states, in which the peripheral elements produce nearly identical behaviors, while the dynamics of the major element is quite different.

We conclude that introducing a major element into a population of oscillators consisting of interacting or noninteracting elements, can give rise to new synchronized states and to increased stability regions for already existing synchronized states in the systems.

\section{Acknowledgment}

I. O. and Yu. M. acknowledge support from the Danish Natural Science Foundation, and from the European Commission (BioSim Network, contract No. 005137).

\section{References}

[1] V. S. Afraĭmovich and V. I. Nekorkin, Chaos of traveling waves in a discrete chain of diffusively coupled maps, Internat. J. Bifur. Chaos Appl. Sci. Engrg. 4 (1994), no. 3, 631-637.

[2] J. C. Alexander, J. A. Yorke, Z. You, and I. Kan, Riddled basins, Internat. J. Bifur. Chaos Appl. Sci. Engrg. 2 (1992), no. 4, 795-813.

[3] O. V. Aslanidi, O. A. Mornev, O. Skyggebjerg, P. Arkhammar, O. Thastrup, M. P. Sørensen, P. L. Christiansen, K. Conradsen, and A. C. Scott, Excitation wave propagation as a possible mechanism for signal transmission in pancreatic islets of Langerhans, Biophys. J. 80 (2001), no. 3, 1195-1209. 
[4] F. M. Atay, Total and partial amplitude death in networks of diffusively coupled oscillators, Phys. D 183 (2003), no. 1-2, 1-18.

[5] V. N. Belykh, I. V. Belykh, N. Komrakov, and E. Mosekilde, Invariant manifolds and cluster synchronization in a family of locally coupled map lattices, Discrete Dyn. Nat. Soc. 4 (2000), no. 3, 245-256.

[6] V. N. Belykh, I. V. Belykh, and E. Mosekilde, Cluster synchronization modes in an ensemble of coupled chaotic oscillators, Phys. Rev. E 63 (2001), 036216.

[7] T. Bohr and O. B. Christensen, Size dependence, coherence, and scaling in turbulent coupled-map lattices, Phys. Rev. Lett. 63 (1989), no. 20, 2161-2164.

[8] R. M. Borisyuk and Y. B. Kazanovich, Oscillatory neural network model of attention focus formation and control, Biosystems 71 (2003), no. 1-2, 29-38.

[9] S. Danø, P. G. Sørensen, and F. Hynne, Sustained oscillations in living cells, Nature 402 (1999), 320-322.

[10] S. De Monte, F. d'Ovidio, and E. Mosekilde, Coherent regimes in globally coupled dynamical systems, Phys. Rev. Lett. 90 (2003), no. 5, 054102.

[11] G. B. Ermentrout, Oscillator death in populations of "all to all" coupled nonlinear oscillators, Phys. D 41 (1990), no. 2, 219-231.

[12] H. Fujisaka and T. Yamada, Stability theory of synchronized motion in coupled-oscillator systems, Progr. Theoret. Phys. 69 (1983), no. 1, 32-47.

[13] J. Jost and M. P. Joy, Spectral properties and synchronization in coupled map lattices, Phys. Rev. E (3) 65 (2002), no. 1, part 2, 016201, 9 pp.

[14] K. Kaneko, Lyapunov analysis and information flow in coupled map lattices, Phys. D 23 (1986), no. 1-3, 436-447.

[15] Clustering, coding, switching, hierarchical ordering, and control in a network of chaotic elements, Phys. D 41 (1990), no. 2, 137-172.

[16] _ Relevance of dynamic clustering to biological networks, Physica D 75 (1994), no. 1-3, $55-73$.

[17] Y. B. Kazanovich and R. M. Borisyuk, Dynamics of neural networks with a central element, Neural Networks 12 (1999), no. 3, 441-454.

[18] _ Object selection by an oscillatory neural network, Biosystems 67 (2002), no. 1-3, 103111.

[19] I. Matskiv and Y. Maistrenko, Synchronization and clustering among two interacting ensembles of globally coupled chaotic oscillators, Proc. 11th International IEEE Workshop on Nonlinear Dynamics of Electronic Systems (NDES '03), Institute of Neuroinformatics, University/ETH Zurich, Scuol, 2003, pp. 165-168.

[20] I. Matskiv, Y. Maistrenko, and E. Mosekilde, Synchronization between interacting ensembles of globally coupled chaotic maps, Phys. D 199 (2004), no. 1-2, 45-60.

[21] J. Milnor, On the concept of attractor, Comm. Math. Phys. 99 (1985), no. 2, 177-195.

[22] E. Mosekilde, Y. Maistrenko, and D. Postnov, Chaotic Synchronization. Applications to Living Systems, World Scientific Series on Nonlinear Science. Series A: Monographs and Treatises, vol. 42, World Scientific, New Jersy, 2002.

[23] E. Ott and J. C. Sommerer, Blowout bifurcations: the occurrence of riddled basins and on-off intermittency, Phys. Lett. A 188 (1994), 39-47.

[24] L. M. Pecora, Synchronization conditions and desynchronizing patterns in coupled limit-cycle and chaotic systems, Phys. Rev. E (3) 58 (1998), no. 1, 347-360.

[25] L. M. Pecora and T. L. Carroll, Master stability functions for synchronized coupled systems, Phys. Rev. Lett. 80 (1998), no. 10, 2109-2112.

[26] A. Pikovsky, O. Popovych, and Y. Maistrenko, Resolving clusters in chaotic ensembles of globally coupled identical oscillators, Phys. Rev. Lett. 87 (2001), no. 4, 044102. 
[27] A. Pikovsky, M. Rosenblum, and J. Kurths, Synchronization. A Universal Concept in Nonlinear Sciences, Cambridge Nonlinear Science Series, vol. 12, Cambridge University Press, Cambridge, 2001.

[28] O. Popovych, Y. Maistrenko, and E. Mosekilde, Loss of coherence in a system of globally coupled maps, Phys. Rev. E 64 (2001), 026205.

[29] _ Role of asymmetric clusters in desynchronization of coherent motion, Phys. Lett. A 302 (2002), no. 4, 171-181.

[30] T. Shimada and K. Kikuchi, Periodicity manifestations in the turbulent regime of the globally coupled map lattice, Phys. Rev. E 62 (2000), no. 3, 3489-3503.

[31] S. H. Strogatz, Exploring complex networks, Nature 410 (2001), no. 6825, 268-276.

[32] S. H. Strogatz and I. Stewart, Coupled oscillators and biological synchronization, Sci. Amer. 269 (1993), no. 6, 102-109.

[33] P. A. Tass, Desynchronizing double-pulse phase resetting and application to deep brain stimulation, Biol. Cybernet. 85 (2001), no. 5, 343-354.

[34] _ Effective desynchronization with a stimulation technique based on soft phase resetting, Europhys. Lett. 57 (2002), no. 2, 164-170.

[35] Stimulus-locked transient phase dynamics, synchronization and desynchronization of two oscillators, Europhys. Lett. 59 (2002), no. 2, 199-205.

[36] K. Wiesenfeld, P. Colet, and S. H. Strogatz, Synchronization transition in a disordered Josephson series array, Phys. Rev. Lett. 76 (1999), no. 3, 404-407.

[37] F. H. Willeboordse, Encoding travelling waves in a coupled map lattice, Internat. J. Bifur. Chaos Appl. Sci. Engrg. 4 (1994), no. 6, 1667-1673.

[38] A. T. Winfree, Biological rhythms and the behavior of populations of coupled oscillators, J. Theoret. Biol. 16 (1967), 15-42.

Iryna Omelchenko: Institute of Mathematics, National Academy of Sciences of Ukraine, 3 Tereshchenkivska Street, 01601 Kyiv-4, Ukraine

E-mail address: matskiv@imath.kiev.ua

Yuri Maistrenko: Institute of Mathematics, National Academy of Sciences of Ukraine, 3 Tereshchenkivska Street, 01601 Kyiv-4, Ukraine

E-mail address: maistrenko@imath.kiev.ua

Erik Mosekilde: Department of Physics, Technical University of Denmark, 2800 Kgs. Lyngby, Denmark

E-mail address: erik.mosekilde@fysik.dtu.dk 


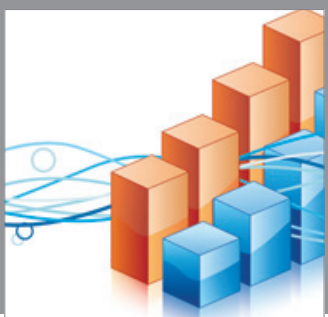

Advances in

Operations Research

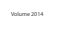

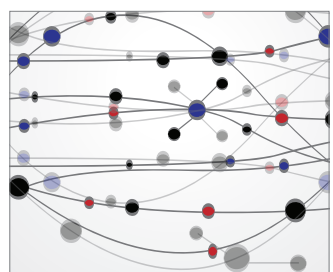

\section{The Scientific} World Journal
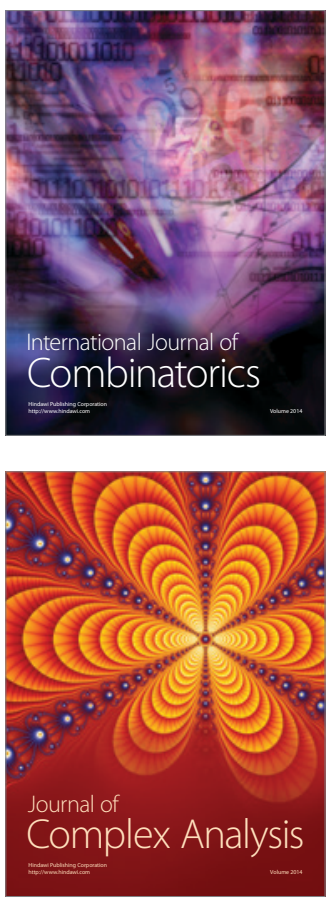

International Journal of

Mathematics and

Mathematical

Sciences
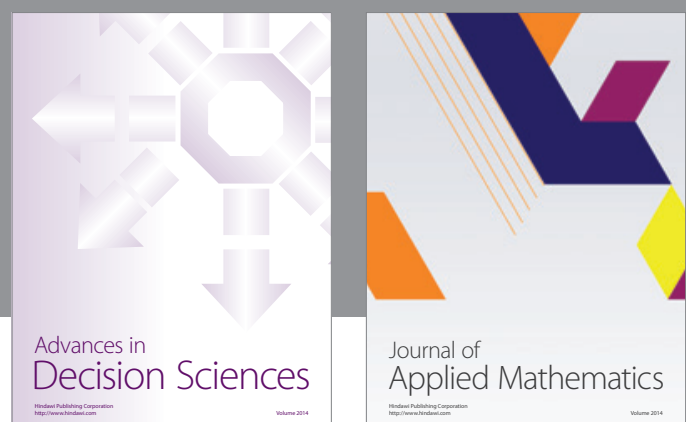

Journal of

Applied Mathematics
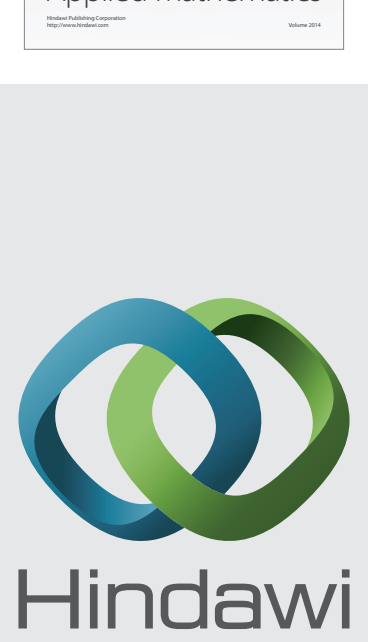

Submit your manuscripts at http://www.hindawi.com
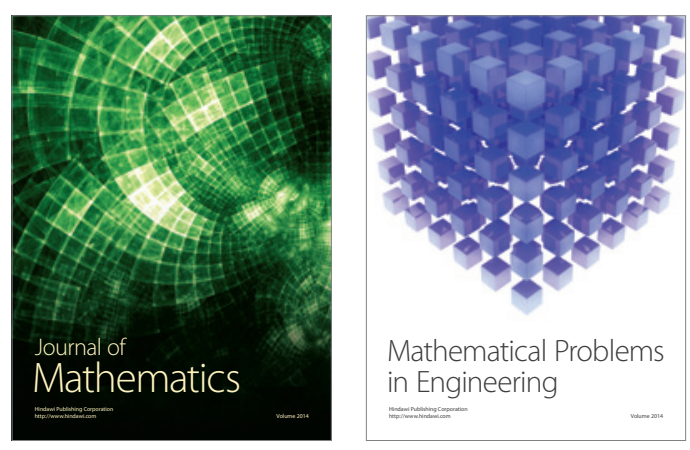

Mathematical Problems in Engineering
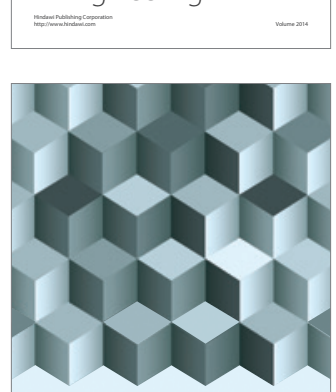

Journal of

Function Spaces
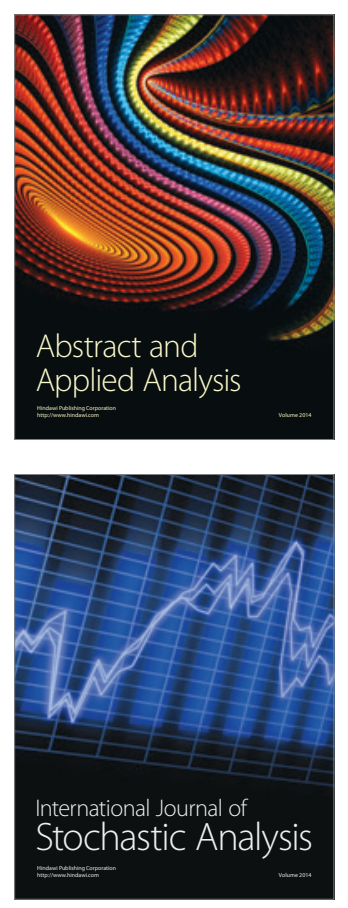

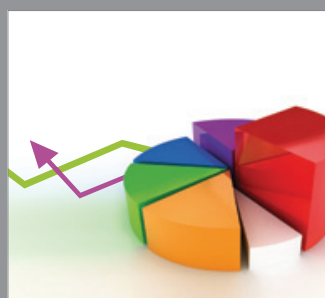

ournal of

Probability and Statistics

Promensencen
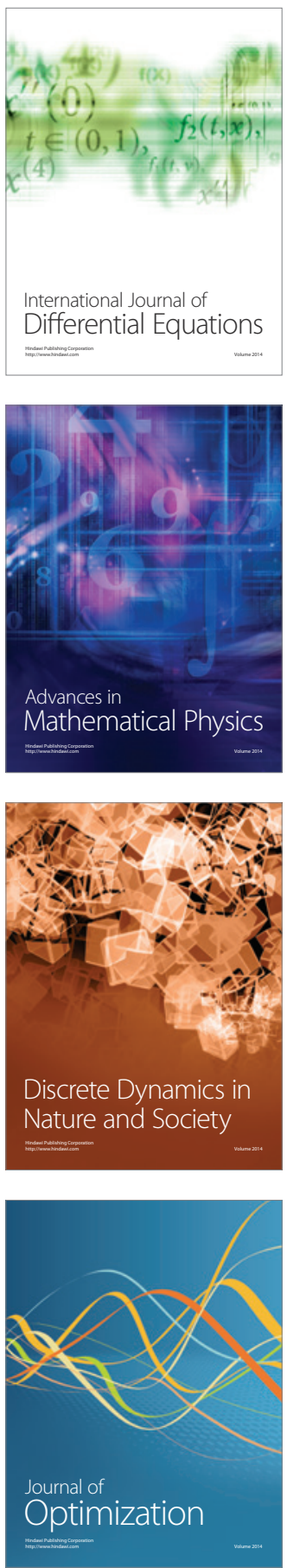